\title{
Research on Calibration Test Method of Infrared Thermal Imager for Power System
}

\author{
Weiwei $Z H_{A N G}{ }^{1, a}{ }^{*}$, Guangke XU ${ }^{1, b}$, Ming $\mathrm{LI}^{1, \mathrm{c}}$, Dongdong $X \mathrm{U}^{1, \mathrm{~d}}$, \\ Fuchun SUN ${ }^{1, e}$, Guangtao WANG ${ }^{1, f}$, Kun WANG ${ }^{2, g}$ \\ ${ }^{1}$ State Grid Shandong Electric Power Research Institute, Jinan, 250002, China \\ ${ }^{2}$ Shandong Zhongshiyitong Group Co., Ltd. Jinan, 250002, China \\ aweiwei.1302@163.com, bxuguangkesd@163.com, ${ }^{\mathrm{c} l i m i n g 88419 @ 163 . c o m,{ }^{d} 491149932 @ q q . c o m,}$ \\ 353373462@qq.com, guangtao35@163.com,359721419@qq.com
}

Keywords: thermal infrared imager, power system, calibration test method, NETD.

Abstract. The power system application of the infrared thermal imager is introduced in this article. By analyzing the special requirements of power system applications, several important performance parameters of the infrared thermal are summed up. This paper presents a more comprehensive calibration test method of infrared thermal imager applied to power system.

\section{Introduction}

The infrared detection diagnosis technology application in power system has a history of more than 20 years in China. In order to improve the safety, economy and reliability of power grid, the infrared thermal imager using the advantages of high efficiency, safety, no power outage has been widely used [1]. With the wide application of infrared thermal imaging technology, the higher request for the performance parameter testing technology of infrared thermal imager is put forward. Infrared thermal imaging testing technology, therefore, must adapt to the development of infrared technology [2].

\section{Analysis of infrared thermal imager for power system}

Currently, the infrared thermal imaging application in the power system is divided into two aspects: the fault diagnosis of power equipment inspection and electrical fire prevention safety testing.

The operation of electrical equipment associated with fever heating up, and the development and formation of the failure is not happen all at once, there have been a process of change, but most of them are closely connected with the heating temperature. These heat anomalies can be found by using direct observation and measurement of the infrared thermal imager. And the location and severity of failure will be mastered. A lot of economic losses can be reduced if the failure and hidden danger are timely processed.

If the electrical equipment run in the state of the electrical hidden dangers of accidents or the electrical fire hazards, the fire source by overheating or electro-discharging will be produced, finally lead to fires. The infrared thermal imaging technology-based means of detection has a significant effect in discovering the hidden danger or monitoring the operating state in electrical equipment and electrical circuit. It can effectively prevent and reduce the electrical fire accident.

The application of thermal imager in power system is mostly through long distance non-contact scanning inspection. The working environment temperature is changeable. The test distance is uncertain. And the electrical equipment has a wide variety type. In this case, it requires infrared thermal imager the measured object surface temperature distribution can be clearly imaged and its temperature accuracy and sensitivity in a high level. The higher requirements of the infrared thermal imager performance are put forward. It needs a more comprehensive and effective detection methods for infrared thermal imager in power system. 


\section{The infrared thermal imager performance testing}

The infrared thermal imager has a number of basic parameters, including temperature measurement accuracy, noise equivalent temperature difference (NETD), character measuring range, consistent measurement duration, effect of ambient temperature, measurement uniformity, minimum resolvable temperature difference (MRTD), electromagnetic compatibility, low voltage electrical safety, environmental adaptability, etc [4]. The electromagnetic compatibility, low voltage electrical safety and environmental adaptability are the general requirements for electrical products. These will not be researched in this article. Minimum resolvable temperature difference (MRTD) reflects the viewer's eye resolution capability to the infrared thermal imager output image. It relates to the observer subjective factors. It will vary with changes in people's differences. So the MRTD will not be researched in this article.

This paper selects temperature measurement accuracy, noise equivalent temperature difference (NETD), character measuring range, consistent measurement duration, effect of ambient temperature, measurement uniformity as the infrared thermal imager calibration test subjects. Because of these performance parameters have a greater influence in power system.

\section{Temperature measurement accuracy}

The temperature measurement accuracy is a basic and most important parameter in the infrared thermal imager. Firstly, select the range of the upper limit, the lower limit and the median as the temperature calibration point. Secondly, put blackbody radiation source at the working distance of 2 $\mathrm{m}$ to $3 \mathrm{~m}$, and set the infrared thermal imager emission rate to 1 . Then, measure the temperature of the blackbody radiation target center [5].

When $\mathrm{t}_{2}<100^{\circ} \mathrm{C}$, according to the equation (1)

$\theta=t_{2}-t_{1}$

When $t_{2} \geqslant 100^{\circ} \mathrm{C}$, according to the equation (2)

$$
\theta=\frac{t_{1}-t_{2}}{t_{2}} \times 100 \%
$$

Where, $\theta$ represents the temperature measurement accuracy, $t_{1}$ represents the known standard blackbody radiation temperature, $\mathrm{t}_{2}$ represents the infrared thermal imager reading temperature.

\section{The noise equivalent temperature difference (NETD)}

NETD is one of the important indicators of thermal imager sensitivity. It directly affects the system's important parameters such as target sensitivity, detection range indicator. NETD is defined as the temperature difference between the blackbody target and background when the ratio of the system reference circuit output signal peak and root mean square noise signal is $1[6,7]$.

$$
N E T D=\frac{\Delta T}{S / N}
$$

Where, $\Delta T$ represents the temperature difference between the target and background, $S$ represents the peak signal level of target signal, $\mathrm{N}$ represents root mean square noise level.

The NETD test block diagram is shown in Fig.1. Firstly, set the blackbody radiation temperature difference as $\Delta T=2 \mathrm{~K}$, put the thermal imager on the distance of $1 \mathrm{~m}$ from the blackbody, and adjust the focus make the target clear imaging. The target pattern must be less than 1/10 of the total field of view. Then measure the peak signal value $S$ by an oscilloscope. Secondly, cover the entrance pupil of the thermal imager, measure the root mean square noise voltage $\mathrm{N}$ by a voltmeter. Finally, take the measured values into the equation (3) to get NETD. 


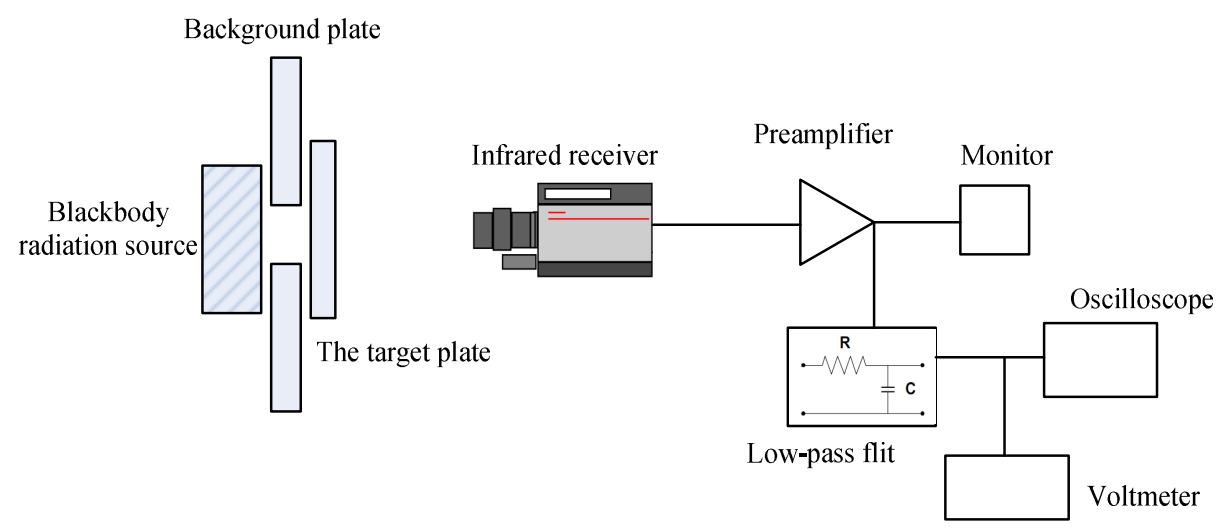

Fig. 1 The NETD test block diagram

\section{The character measuring range}

Set two blackbody radiation source temperature $0{ }^{\circ} \mathrm{C}$ and $100{ }^{\circ} \mathrm{C}$ respectively. Place the infrared thermal imager in $2 \mathrm{~m}$ distance of the blackbody. Make the two blackbody in the same field of view. The infrared thermal imager uses the same optical lens, do not change the light bar, and do not increase in filter. Then observe the thermal imager imaging results and measure temperature at the same time. It requires clear imaging and the temperature measurements meeting the temperature measurement accuracy [5].

\section{The consistent measurement duration}

The consistent measurement duration is very important for working long hours' thermal imager. It proves the stability of thermal imager in the test time.

Set the blackbody radiation temperature at $50^{\circ} \mathrm{C}$. Make the thermal imager can clear imaging within the specified working distance. In the absence of human intervention, read the infrared temperature point data once every 10 minutes. The general test time of off-line thermal imager is 2 hours. The temperature measurements require meeting the temperature measurement accuracy.

\section{The effect of ambient temperature}

The effect of ambient temperature is to test the accurately measuring temperature ability of thermal imager when temperature changes. Set blackbody radiation temperature at any temperature within the temperature range of the thermal imager. Put the thermal imager into the $40^{\circ} \mathrm{C}$ incubator for 1 hour. Get it out and put it at room temperature for 15 minutes. Then measure the temperature of blackbody, and measure a second time after 15 minutes. Then Put the thermal imager into the $0^{\circ} \mathrm{C}$ incubator for 1 hour. Repeat the process when it is stable. The temperature measurements require meeting the temperature measurement accuracy [8].

\section{The measurement uniformity}

The temperature distribution reliability provided by the thermal imager is largely depending on the measurement uniformity. Take the most central parts of the region thermal imager as a reference, which divide the monitors imaging screen into 9 areas. As shown in Fig. 2. Set the blackbody radiation temperature to $100^{\circ} \mathrm{C}$, and place thermal imager in a predetermined working distance. Adjust the thermal imager to make it clear imaging, and the blackbody radiation image can be filled with the field of view. Select the center point from 1 to 9 regions to measure the temperature. And obtain temperature measurements $t_{i}(i=1,2,3 \ldots 9)$ [5]. 


\begin{tabular}{|c|c|c|}
\hline 1 & 2 & 3 \\
\hline 4 & 5 & 6 \\
\hline 7 & 8 & 9 \\
\hline
\end{tabular}

Fig. 2 Monitor imaging screen

When $\mathrm{t}_{\mathrm{i}}<100^{\circ} \mathrm{C}$, according to the equation (4)

$\phi=t_{5}-t_{i}$

When $t_{i} \geqslant 100^{\circ} \mathrm{C}$, according to the equation (5)

$\phi=\frac{t_{\mathrm{i}}-t_{5}}{t_{5}} \times 100 \%$

Where, $\phi$ represents the measurement uniformity, $t_{i}$ represents the temperature measurement in thermal imager of each area, $t_{2}$ represents the temperature measurement in thermal imager of the 5 area.

\section{Conclusions}

Through the analysis of the thermal imager application in power system obtained several important performance parameters of thermal imager. These performance parameters calibration testing methods are specific researched in this article. Provide a reference for more comprehensive assessment of thermal imager in power system.

\section{References}

[1] Jian Yang, DING Na. Analyzing electrical trouble by an infrared thermal imaging system, J. Electric Switcher, 2013, 50(1):101-103. (In Chinese)

[2] Yunhong Li, Xiaogang Sun, Jihong Lian. Research on performance testing of thermal infrared imaging system, J. Infared and Laser Engineering, 2008,S2:458-462. (In Chinese)

[3] Jixian Ma, Qing Yang, Liang Guo, Ming Lv, Lifang Xie. Research on promoting the accuracy of electrical equipment diagnosing with infrared detection, J. North China Electric Power, 2012,08:55-60. (In Chinese)

[4] Hongyu Su, Xianliang Zhang, Yu Chen. Characteristics evaluation of infrared thermal imaging system, J. China Measurement \& Test,2010,01:14-19. (In Chinese)

[5] GB/T 19870-2005<Industrial insperting thermal imagers>. (In Chinese)

[6] Tieli Hu, Shibang Ma, Yu Guo. Spatial NETD measurement of thermal imager, J. Journal of Applied Optics, 2014,06:1094-1098. (In Chinese)

[7] Chengbin An, Yin Wang. Analysis of NETD test for thermal imaging system, J. Infrared and Laser Engineering, 2010,03:414-417. (In Chinese)

[8] DL/T664-2008<Application rules of infrared diagnasis for live electrical equipment>. (In Chinese) 